Marin H. Kollef

Cristina Vazquez Guillamet

\section{If antibiotics did not exist}

Received: 21 October 2014

Accepted: 23 October 2014

Published online: 11 November 2014

(C) Springer-Verlag Berlin Heidelberg and

ESICM 2014

\author{
M. H. Kollef (区) \\ Division of Pulmonary and Critical Care \\ Medicine, Washington University School of \\ Medicine, 660 South Euclid Avenue, \\ Campus Box 8052, St. Louis, MO 63110, \\ USA \\ e-mail: mkollef@dom.wustl.edu \\ Tel.: (314) 454-8764
}

\author{
C. V. Guillamet \\ Division of Pulmonary Critical Care and \\ Sleep Medicine, University of New Mexico \\ School of Medicine, Albuquerque, \\ NM, USA \\ C. V. Guillamet \\ Division of Infectious Diseases, University \\ of New Mexico School of Medicine, \\ Albuquerque, NM, USA
}

It is interesting to speculate on what the world would be like if antibiotics did not exist. The most striking findings would be deadly infections caused by uniformly sensitive but virulent bacteria contrasted with very few if any infections caused by "modern medicine" pathogens such as MRSA (methicillin-resistant Staphylococcus aureus), MDR-GNRs (multidrug-resistant gram-negative rods), Clostridium difficile and Candida spp. Take for example infections caused by Streptococcus pneumoniae, Neisseria meningitides, Haemophilus influenzae and Staphylococcus aureus. These represent common causes of community-acquired infections in both adults and children including some that can be quite serious such as pneumonia, bacteremia and meningitis. The development of penicillins and sulfonamides at the beginning of the twentieth century turned these previously fatal illnesses into conditions amenable to treatment allowing the host to recover. However, over decades of antibiotic use, and often abuse due to inappropriate indications, resistance developed to many of the antibiotic classes previously employed for their treatment to include penicillins, macrolides and fluoroquinolones.

Most would argue that antibiotics have improved the overall global environment for mankind by allowing treatment of bacterial infections. However, the introduction of antibiotics was rapidly followed by emergence of resistance-it took Staphylococcus aureus 1 year to become resistant to methicillin after its introduction in 1960 - which has now resulted in the development of new strains of antibiotic-resistant "superbugs" that almost certainly would not have emerged without exposure to these agents. Moreover, at the beginning of the twentieth century several socioeconomic influences occurred that had a dramatic impact on the use of antibiotics and the subsequent development of antimicrobial resistance. Mass production of antibiotics and the availability of oral antibiotics greatly enhanced exposure to these agents outside of the hospital setting and allowed their widespread use in the agricultural industry. These changes overlapped with greater expectations from patients and their families to receive a "magic bullet" to cure their illness or infection. Only in the last several decades have we recognized that many of the prescribed antibiotics have been used to treat nonbacterial infections such as viral bronchitis and otitis, thus needlessly exposing patients to potential side effects of these drugs while increasing the burden of antibiotic resistance.

Another important factor promoting greater antimicrobial consumption has been the changing spectrum of patients and the institutions where they are cared for. Patients are older, more immunocompromised because of treatments aimed at underlying malignancies, autoimmune disorders and transplants, and have acquired diseases not present at the turn of the century such as 
acquired immunodeficiency syndrome. Additionally, hospitals have developed highly specialized units to care for critically ill individuals as well as those with specialized conditions. This has contributed to the grouping, and in some cases crowding, of individuals receiving multiple prophylactic and empiric antibiotic regimens often for prolonged periods of time. These types of factors have led to outbreaks and in some cases dissemination of antibiotic-resistant "superbugs" to include vancomycinresistant enterococci, carbapenem-resistant (and now coexistent colistin-resistant) Enterobacteriaceae and extremely drug-resistant nonfermenters such as Pseudomonas aeruginosa, Acinetobacter species and Stenotrophomonas maltophilia. These "superbugs," sometimes identified as the "ESKAPE" organisms (Enterococcus faecium, MRSA, Klebsiella pneumoniae, Acinetobacter, Pseudomonas, Enterobacteriaceae), are often normal colonizers, also able to persist in the environment and capable of transferring resistance elements among species. For instance, the not so virulent Acinetobacter has become panresistant at times after gradually accumulating resistance elements.

We are now in a world that has to grapple with infections caused by antibiotic-resistant bacteria in both the community setting as well as the healthcare setting. The latter is especially problematic as many patients, especially toward the end of their lives, spend increasingly greater amounts of time in hospitals, nursing homes and other facilities where they are often empirically treated with antibiotics and exposed to other nosocomial bacteria due to inadequate infection control practices. This has resulted in a vicious circle whereby broader and broader antibiotics are prescribed because of subsequent colonization and infection with more antibiotic-resistant microorganisms. The situation has become so dire that global organizations, including the World Health Organization, the Centers for Disease Control and Prevention, the European Centre for Disease Prevention and Control, and the World Alliance against Antibiotic Resistance, have made combating bacterial resistance one of the top priorities for improving the health of the world's inhabitants.

Battling antibiotic resistance, including the clinical and societal impact of this problem, will require a dedicated and multifaceted approach. We will need concerted efforts to decrease the antibiotic burden not only in hospital settings but also in outpatient clinics, nursing homes and the agricultural industry. Fortunately, strategies are emerging that should slow and possibly reverse some of the trends seen over the past century in terms of resistance emergence. The concept of antibiotic de-escalation has arisen as an important component of antimicrobial stewardship. Many studies have demonstrated the efficacy and safety of performing de-escalation based on microbiology results. Moreover, prediction scores and algorithms have emerged to guide clinicians as to when antibiotics, and more specifically broad-spectrum antibiotics, should be empirically administered or withheld. At the same time, biomarkers such as procalcitonin [1, 3], $\beta$-D-glucan and interleukin-18 are now available to help direct the use of antimicrobials in patients with suspected infections. One other area that could easily be tackled is shortening the courses of antibiotics. In randomized trials shorter courses of antibiotics have yielded similar outcomes to longer courses even in serious infections such as ventilatorassociated pneumonia. Unfortunately, none of these approaches is foolproof. All have the potential to allow inadequate treatment of an underlying infection or overuse of antibiotics because of their limited accuracy.

Much hope has been placed in antimicrobial stewardship programs to combat antimicrobial resistance. The current literature is indicative but not conclusive of decreased levels of resistance when using such programs. Moreover, the exact methodology for stewardship remains to be determined since empowering the use of certain antibiotics over others may just lead to "squeezing of the balloon" (decreased resistance to the nonformulary antibiotic and increased resistance to the antibiotic most commonly used). Mathematical models seem to suggest lower resistance with more heterogeneous antibiotic administration. The development of new antibiotics along with the emergence of rapid diagnostics for the identification of specific pathogens as well as their antibiotic susceptibility holds promise for improving the management of, and potentially reducing the prevalence of, antibiotic-resistant infections.

Within the next 3-5 years new agents directed against gram-negative bacteria will become available including carbavance, ceftolozane-tazobactam, ceftazidime-avibactam, plazomicin, eravacycline, relebactam, brilacidin, BAL30072, aztreonam-avibactam, carbapenems with ME 1071 and S-649266, a novel siderophore cephalosporin. These agents will provide enhanced activity against $\beta$ lactamase producers, carbapenem-resistant bacteria and in some cases even metallo- $\beta$-lactamase-producing bacteria. Although resurgence in the antibiotic pipeline has been noted after an initial decline (approximately four new drugs approved yearly in the 1980 s vs. one drug per year in the 2000s), the worry persists that indiscriminate use of novel antibiotics will result in the development of resistance to them. The challenge to clinicians is to develop strategies that will optimize the use of these new drugs while preventing resistance emergence. Rapid diagnostics may hold the key to accomplishing this important balance. A number of novel methods aimed at rapidly identifying pathogens and their susceptibilities, typically within 4-6 h, are available, including molecular methods [e.g., polymerase chain reaction, mass spectroscopy techniques (MALDI-TOF-matrix-assisted laser desorption/ionization time of flight)] and advanced automated microscopy techniques.

To summarize, the advent of antibiotic therapy has clearly led to improvements in, and the saving of, 
countless individuals. At the same time, antibiotic usage and abuse has resulted in the current situation where extremely drug-resistant bacteria have brought us back to a "pre-antibiotic era." Going forward, we must insure that the management of these serious antibiotic-resistant infections occurs in a manner that is sustainable for the long run. This will mean disciplined use of antibiotics, utilization of alternative nonantibiotic therapies such as vaccines and monoclonal antibodies, and the incorporation of cost-effective rapid diagnostic methods.

Conflicts of interest Dr. Kollef's efforts were supported by the Barnes-Jewish Hospital Foundation.

\section{References}

1. Kollef MH, Micek ST (2014) Rational use of antibiotics in the ICU: balancing stewardship and clinical outcomes. JAMA 312:1403-1404. doi:10.1001/jama.2014.8427

2. Timsit JF, Harbarth S, Carlet J (2014) De-escalation as a potential way of reducing antibiotic use and antimicrobial resistance in ICU. Intensive Care Med 40:1580-1582. doi:10.1007/s00134-014-3485-3

3. Nathan C, Cars O (2014) Antibiotic resistance-problems, progress, and prospects. N Engl J Med 371(19):1761-1763

4. Spellberg B, Bonomo RA (2014) The deadly impact of extreme drug resistance in Acinetobacter baumannii. Crit Care Med 42:1289-1291. doi:10.1097/CCM.0000000000000181

5. Rossi F, Diaz L, Wollam A, Panesso D, Zhou Y, Rincon S, Narechania A, Xing G (2014) Transferable vancomycin resistance in a community-associated MRSA lineage. N Engl J Med. doi:10.1056/NEJMoa1303359

6. Kuehn BM (2014) CDC: Hospital antibiotic use promotes resistance: checklist can improve practices. JAMA 311:1485-1486. doi:10.1001/jama.2014.2847
7. Burnham CA, Frobel RA, Herrera ML, Wickes BL (2014) Rapid ertapenem susceptibility testing and Klebsiella pneumoniae carbapenemase phenotype detection in Klebsiella pneumoniae isolates by use of automated microscopy of immobilized live bacterial cells. J Clin Microbiol 52:982-986.

doi:10.1128/JCM.03255-13

8. Laffler TG, Cummins LL, McClain CM, Quinn CD, Toro MA, Carolan HE, Toleno DM, Rounds MA, Eshoo MW, Stratton CW, Sampath R, Blyn LB, Ecker DJ, Tang YW (2013) Enhanced diagnostic yields of bacteremia and candidemia in blood specimens by PCR-electrospray ionization mass spectrometry. J Clin Microbiol 51:3535-3541.

doi:10.1128/JCM.00876-13

9. European Centre for Disease Prevention and Control/European Medicines Agency (2009) ECDC/EMEA Joint Technical Report. The bacterial challenge: time to react
10. Infectious Diseases Society of America2004Bad bugs. A public health crisis brews, No drugs. as antibiotic discovery stagnates

11. Rice LB (2008) Federal funding for the study of antimicrobial resistance in nosocomial pathogens: no ESKAPE. J Infect Dis 197:1079-1081. doi:10.1086/533452

12. Meyer E, Gastmeier P, Deja M, Schwab F (2013) Antibiotic consumption and resistance: data from Europe and Germany. Int J Med Microbiol 303:388-395. doi:10.1016/j.ijmm.2013.04.004

13. Davey P, Brown E, Fenelon L, Finch R, Gould I, Holmes A, Ramsay C, Taylor E, Wiffen P, Wilcox M (2006) Systematic review of antimicrobial drug prescribing in hospitals. Emerg Infect Dis 12:211-216 